\title{
Synthesis of 8-bromoisoquinolines and a crystal structure of an acyclic secondary amine-borane
}

\author{
Montserrat Armengol, Madeleine Helliwell ${ }^{\#}$, and John A. Joule* \\ Chemistry Department, The University of Manchester, Manchester M13 9PL, U.K. \\ phone: 44 (0) 161275 4633; fax: 44 (0) 1612754598 \\ EE-mail: j.a.joule@man.ac.uk \\ ${ }^{* *}$ E-mail: mad@pec6.sc.ch.man.ac.uk
}

Dedicated to Professor Otto Meth-Cohn on the occasion of his $65^{\text {th }}$ birthday (received 12 Sep 00; accepted 03 Oct 00; published on the web 11 Oct 00)

\begin{abstract}
8-Bromo-7-methoxyisoquinoline was produced by Jackson's modification of the Pomeranz-Fritsch ring synthesis accompanied by 8-bromo-3-(8-bromo-7-methoxyisoquinolin-4-yl)-1,2,3,4tetrahydro-7-methoxy-2-(4-methylphenylsulfonyl)isoquinoline. A mechanism for the formation of the latter is suggested. The ready formation of secondary amine- $\mathrm{BH}_{3}$ complexes was noted and the X-ray crystal structure of $N$-(2-bromo-3-methoxybenzyl)aminoacetaldehyde dimethyl acetal boranedetailed.
\end{abstract}

Keywords: 8-Bromoisoquinolines, dimerisation during Pomeranz-Fritsch synthesis, X-ray crystal structure of a secondary amine borane complex

\section{Introduction}

Our interest in the fusion of a third ring to an isoquinoline framework, across the 8- and 1-positions, led to a requirement for 8 -bromoisoquinoline or a derivative, so that a chain could be attached at the 8-position via a coupling procedure. 8-Bromoisoquinoline appears in the literature three times: it was prepared $^{1}$ from isoquinoline by nitration, reduction, diazotisation and Sandmeyer reaction, it was used ${ }^{2}$ to prepare $8,8^{\prime}$-biisoquinoline without mention of its method of synthesis, and it was prepared in $31 \%$ yield by ring synthesis $;^{3}$ we chose to follow the ring synthesis route.

\section{Results and Discussion}

Reaction of 2-bromobenzaldehyde with 2,2-dimethoxyethanamine gave the Schiff base 1, borohydride reduction of which produced the 2-bromobenzylamine-acetal 2 (see later for further discussion of this reduction). Conversion to a tosylamide 3 so that Jackson's modification ${ }^{4}$ of the Pomeranz-Fritsch 


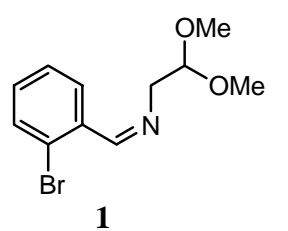<smiles>COC(CNCc1ccccc1Br)OC</smiles><smiles>COC(C[NH3+])Cc1ccccc1Br</smiles><smiles>Brc1cccc2c1C[N+]([Sb])=CC2</smiles><smiles>Brc1cccc2ccncc12</smiles>

isoquinoline ring synthesis could be examined was unrewarding - the expected cyclised product 4 was obtained under strongly acidic conditions, but could not be properly characterised and certainly was not converted to the aromatic isoquinoline under the standard conditions. ${ }^{4}$ Treatment of 1 with a mixture of phosphorus pentoxide and concentrated sulfuric acid, as described ${ }^{3}$ gave only hydrolysis product, however comparable treatment of the benzylamine 2 did produce 8bromoisoquinoline 5 but at best in $6 \%$ yield.

Since our ultimate plans were unaffected by the presence of additional substituents on the isoquinoline benzene ring, we turned to the prospect of ring synthesising 8-bromo-7methoxyisoquinoline ${ }^{5}$ hoping that an electrophilic ring closure would be more efficient at the position para to the methoxy group. 8-Bromo-7-methoxyisoquinoline has been prepared from 7methoxy-8-nitroisoquinoline ${ }^{6}$ by nitro group reduction, diazotisation, and then Sandmeyer reaction ${ }^{7}$ however this would have required a ring synthesis of 7-methoxyisoquinoline.

2-Bromo-3-methoxybenzaldehyde has been synthesised from 2-bromo-3-methoxytoluene by side-chain dibromination then hydrolysis ${ }^{8}$ and more recently by Meyers via introduction of the halogen after lithiation of 3-methoxybenzaldehyde. ${ }^{9}$ Aldehyde from the Meyers route was condensed with 2,2-dimethoxyethanamine giving 6, borohydride reduction then affording the benzylamine-acetal 7 (see later for further discussion of this reduction).<smiles>COc1cccc(/C=N\CC(OC)OC)c1Br</smiles><smiles>COc1cccc(CNCC(OC)OC)c1Br</smiles>

6<smiles>COc1cccc(C[NH+](S)CC(OC)OC)c1Br</smiles><smiles>COc1ccc2ccncc2c1Br</smiles>

After $N$-tosylation producing 8 , heating with $p$-toluenesulfonic acid in toluene at reflux gave the desired 7-methoxy-8-bromoisoquinoline 9 in $19 \%$ yield, together with $27 \%$ of a byproduct with a molecular weight of 630 corresponding to a molecular formula $\mathrm{C}_{27} \mathrm{H}_{24} \mathrm{Br}_{2} \mathrm{~N}_{2} \mathrm{O}_{4} \mathrm{~S}$.<smiles>COc1cc2c(cc1Br)-c1ccc(OC)c(Br)c1C[NH+](S)C2</smiles>

10 
To this byproduct we assign structure 10, the key spectroscopic features leading to this assignment, being ${ }^{1} \mathrm{H}-\mathrm{NMR}$ signals for two geminally coupled methylene groups $(\delta 4.25 \& 4.73)$ and $(\delta 3.27 \&$ $3.87)$ the latter further coupled to a methine proton $(\delta 5.00)$, and singlet signals $(\delta 9.50$ and $\delta 8.10)$ for two pyridine ring protons at shifts typical for 1- and 3-positions of an aromatic isoquinoline. We envisage the formation of 10 involving ring closure to enamine-tosylamide 11 which then serves two roles: it is protonated to generate iminium species 12 which is then attacked by a second molecule of 11 giving 13 and following losses of proton ( ${ }^{\circledR}$ 14) and toluenesulfinic acid, the observed product 10 is generated (Scheme 1).

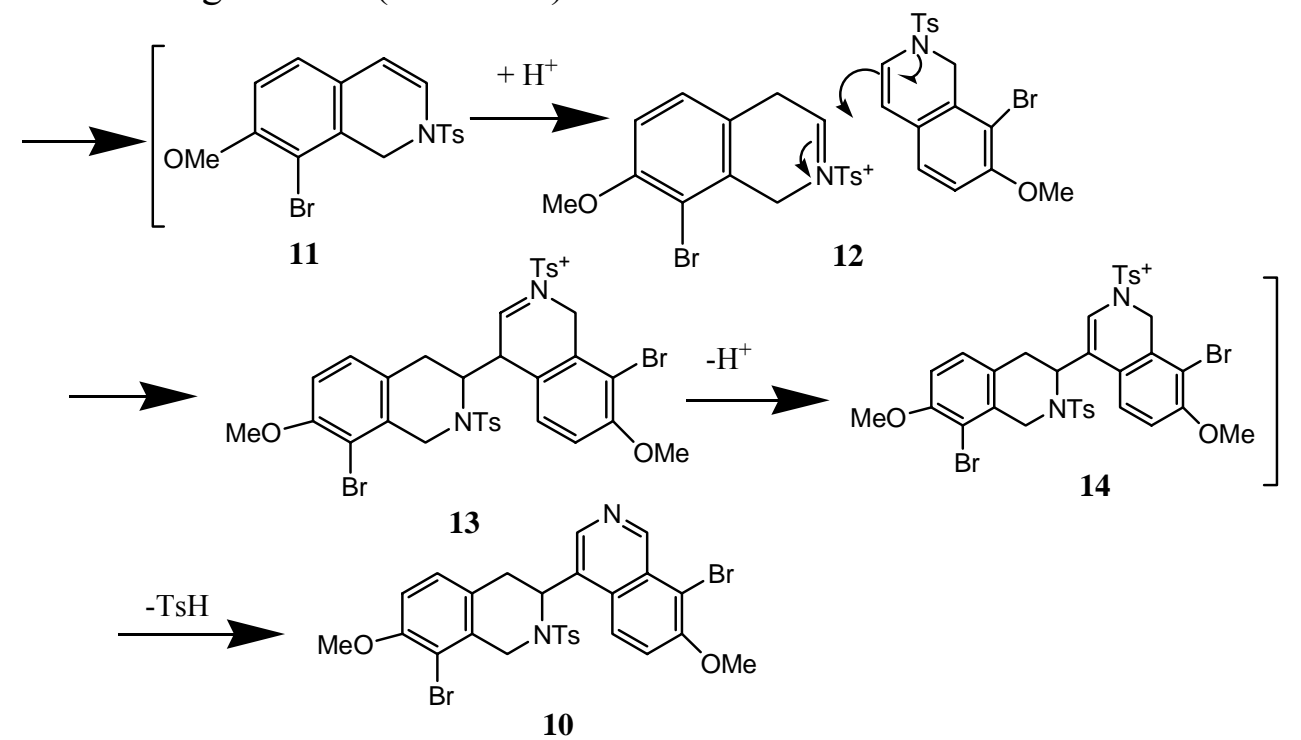

\section{Scheme 1}

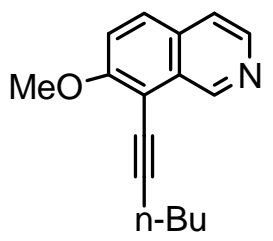

15

In a test coupling it was shown that 8-bromoisoquinoline 9 reacted in modest yield with hex-1-yne to produce the alkynylisoquinoline 15 .

In each of the borohydride reductions of imines 1 and 6, byproducts which could be easily separated by chromatography from the product amines, were obtained. The ${ }^{1} \mathrm{H}-\mathrm{NMR}$ spectra of these byproducts were much more complex than those of the amines and it was not until we realised that these substances were the amine-boranes 16 and 17 now having a chiral centre at the nitrogen that explanations for the complexity became clear - the hydrogen atoms on the adjacent carbon are now distereotopic. To further confirm the assigned structures, each of the amines was reacted with borane in THF to produce the amine-boranes, quantitatively. ${ }^{10}$

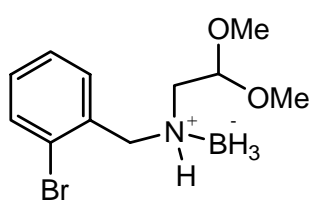

16<smiles>[BH3-][NH+](Cc1cccc(OC)c1Br)CC(OC)OC</smiles>

17

Figure 1. Chem3D drawing of X-ray structure of amine-borane 17. 
Finally, a crystal structure determination on 17 revealed the linked, tetrahedral nitrogen and boron atoms. A Chem3D drawing of 17 made using the X-ray co- ordinates, is shown in Figure 1.

A literature search revealed that this is the first example of a crystal structure of an acyclic secondary amine-borane, involving $\mathrm{BH}_{3}$ itself. ${ }^{11}$ We wondered whether the ready formation of these acyclic amine-boranes was related to the structural complexity of the examples described above. Accordingly, ethanamine was condensed with 2-bromobenzaldehyde, the resulting imine 18 reduced with borohydride generating a mixture of the amine 19 and its borane derivative 20. The amine and its borane were separated and it was again shown that this simple amine reacts quantitatively with borane in THF to form 20. It is noteworthy that for this amine/amine-borane pair, and for the two discussed above, in each case the zwitterionic compound runs faster on chromatography.

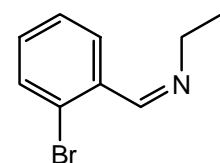

18

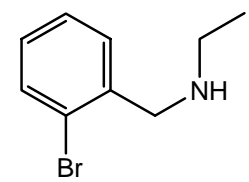

19

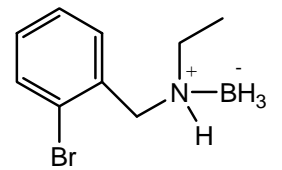

20

\section{Experimental Section}

$N$-(2-Bromobenzylidene)aminoacetaldehyde dimethyl acetal (1). 2-Bromobenzaldehyde (3.4 ml, $29.2 \mathrm{mmol})$ was dissolved in dry toluene $(100 \mathrm{ml})$ and aminoacetaldehyde dimethyl acetal $(3.3 \mathrm{ml}$, $30.6 \mathrm{mmol}$ ) was added. The solution was refluxed under a Dean-Stark trap for 4 hours. The solvent was evaporated in vacuum to give the $N$-(2-bromobenzylidene)aminoacetaldehyde dimethyl acetal 1 (7.6 g, 96\%) as a yellow oil; ${ }^{1} \mathrm{H}-\mathrm{NMR}\left(300 \mathrm{MHz}, \mathrm{CDCl}_{3}\right): \delta 3.46(6 \mathrm{H}, \mathrm{s}), 3.86(2 \mathrm{H}, \mathrm{dd}, J=1.2$ and $5.3 \mathrm{~Hz}), 4.73(1 \mathrm{H}, \mathrm{t}, J=5.3 \mathrm{~Hz}), 7.33(2 \mathrm{H}, \mathrm{m}), 7.59(1 \mathrm{H}, \mathrm{dd}, J=1.4$ and $7.8 \mathrm{~Hz}), 8.05(1 \mathrm{H}, \mathrm{dd}, \mathrm{J}=1.9$ and $7.7 \mathrm{~Hz}), 8.69(1 \mathrm{H}, \mathrm{bs}) ;{ }^{13} \mathrm{C}-\mathrm{NMR}\left(300 \mathrm{MHz} \mathrm{CDCl}_{3}\right): \delta 53.9,63.3,103.5,125.0,127.4,128.2$, 128.7, 131.8, 132.9, 162.4; MS m/z (CI): $274\left(\mathrm{MH}^{+},{ }^{81} \mathrm{Br}, 90 \%\right), 272\left(\mathrm{MH}^{+},{ }^{79} \mathrm{Br}, 100 \%\right)$ (Calcd. for: $\mathrm{C}_{11} \mathrm{H}_{14}{ }^{79} \mathrm{BrNO}_{2}+\mathrm{H}: 272.0287$; HRMS: found: $\left.(\mathrm{M}+\mathrm{H})^{+}, 272.0288\right)$.

N-(2-Bromobenzyl)aminoacetaldehyde dimethyl acetal 2 and N-(2Bromobenzyl)aminoacetaldehyde dimethyl acetal borane (16). To a solution of $N-(2-$ bromobenzylidene)aminoacetaldehyde dimethyl acetal $1(8 \mathrm{~g}, 29.4 \mathrm{mmol})$ in a mixture of THF $(60 \mathrm{ml})$, isopropanol $(60 \mathrm{ml})$, and water $(30 \mathrm{ml}), \mathrm{NaBH}_{4}(6.7 \mathrm{~g}, 176.4 \mathrm{mmol})$ was added and the mixture stirred at room temperature for $2 \mathrm{~h}$. The organic solvents were then evaporated in vacuum. Addition of water and $\mathrm{CH}_{2} \mathrm{Cl}_{2}$ followed by separation, drying, and evaporation of the organic phase under reduced pressure gave a yellow oil. Purification by column chromatography over silica gel eluting with $\mathrm{CH}_{2} \mathrm{Cl}_{2}$ gave the pure amine-borane $16(2.1 \mathrm{~g}, 25 \%)$ as a colourless oil; IR (NaCl): vmax $2376 \mathrm{~cm}^{-1}$; ${ }^{1} \mathrm{H}-\mathrm{NMR}\left(300 \mathrm{MHz}, \mathrm{CDCl}_{3}\right): \delta 2.67(1 \mathrm{H}, \mathrm{m}), 2.78(1 \mathrm{H}, \mathrm{m}), 3.39(3 \mathrm{H}, \mathrm{s}), 3.41(3 \mathrm{H}, \mathrm{s})$, $3.77(1 \mathrm{H}, \mathrm{dd}, J=9.4$ and $13.8 \mathrm{~Hz}), 4.31(1 \mathrm{H}, \mathrm{dd}, J=4.1$ and $13.8 \mathrm{~Hz}), 4.5(1 \mathrm{H}, \mathrm{bs}), 4.85(1 \mathrm{H}, \mathrm{dd}, J=$ 3.8 and $8.1 \mathrm{~Hz}), 7.32(3 \mathrm{H}, \mathrm{m}),(1 \mathrm{H}, \mathrm{d}, J=7.7 \mathrm{~Hz}) ;{ }^{13} \mathrm{C}-\mathrm{NMR}\left(300 \mathrm{MHz}, \mathrm{CDCl}_{3}\right): \delta 54.2,55.3,55.6$, 60.6, 111.0, 124.5, 127.7, 130.1, 132.9, 133.0, 133.3; MS m/z (CI): $288\left(\mathrm{MH}^{+},{ }^{81} \mathrm{Br}, 100 \%\right), 286$ $\left(\mathrm{MH}^{+},{ }^{79} \mathrm{Br}\right.$, 95\%) (Calcd. for: $\mathrm{C}_{11} \mathrm{H}_{19} \mathrm{~B}^{79} \mathrm{BrNO}_{2}$ : M, 287.0697; HRMS: found: $\mathrm{M}^{+}$287.0697). Eluting with $\mathrm{CH}_{2} \mathrm{Cl}_{2} / \mathrm{MeOH}(9: 1)$ gave the amine $2(5.4 \mathrm{~g}, 68 \%)$ as a colourless oil; ${ }^{1} \mathrm{H}-\mathrm{NMR}$ (300 $\left.\mathrm{MHz}^{\mathrm{CDCl}}{ }_{3}\right): \delta 2.78(2 \mathrm{H}, \mathrm{d}, J=5.5 \mathrm{~Hz}), 3.40(6 \mathrm{H}, \mathrm{s}), 3.92(2 \mathrm{H}, \mathrm{s}), 4.53(1 \mathrm{H}, \mathrm{t}, J=5.5 \mathrm{~Hz}), 7.15(1 \mathrm{H}$, $\mathrm{dt}, J=1.7$ and $7.7 \mathrm{~Hz}), 7.32(1 \mathrm{H}, \mathrm{m}), 7.42(1 \mathrm{H}, \mathrm{dd}, J=1.7$ and $7.7 \mathrm{~Hz}), 7.57(1 \mathrm{H}, \mathrm{dd}, J=1.1$ and 8.1 $\mathrm{Hz}) ;{ }^{13} \mathrm{C}-\mathrm{NMR}\left(300 \mathrm{MHz}, \mathrm{CDCl}_{3}\right): \delta 50.1,53.5,53.7,103.7,123.8,127.3,128.5,130.1,132.7$, 
138.9; MS m/z (CI): $276\left(\mathrm{MH}^{+},{ }^{81} \mathrm{Br}, 80 \%\right), 274\left(\mathrm{MH}^{+},{ }^{79} \mathrm{Br}, 100 \%\right)$ (Calcd. for: $\mathrm{C}_{11} \mathrm{H}_{16}{ }^{79} \mathrm{BrNO}_{2}: \mathrm{M}$, 273.0365; HRMS found: $\left.\mathrm{M}^{+}, 273.0360\right)$.

$N$-(2-Bromobenzyl)- $N$-(4-methylphenylsulfonyl)aminoacetaldehyde dimethyl acetal (3). Benzylamino-acetal $2(500 \mathrm{mg}, 1.8 \mathrm{mmol})$ was dissolved in dry pyridine $(2 \mathrm{ml})$ and tosyl chloride (381 mg, $2 \mathrm{mmol}$ ) in dry pyridine $(2 \mathrm{ml}$ ) was added. The solution was stirred at $\mathrm{rt}$ for $24 \mathrm{~h}$. The pyridine hydrochloride was removed by filtration. The filtrate was poured into water and product extracted into ether which was then washed with $1 \mathrm{M}$ aq. $\mathrm{HCl}$, then water. The organic layer was separated, dried, and evaporated under reduced pressure to give the pure tosylamide 3 (770 mg, $88 \%$ ) as a yellow oil; ${ }^{1} \mathrm{H}-\mathrm{NMR}\left(300 \mathrm{MHz}, \mathrm{CDCl}_{3}\right): \delta 2.47(3 \mathrm{H}, \mathrm{s}), 3.23(6 \mathrm{H}, \mathrm{s}), 3.33(2 \mathrm{H}, \mathrm{d}, J=5.3$ $\mathrm{Hz}), 4.38(1 \mathrm{H}, \mathrm{t}, J=5.3 \mathrm{~Hz}), 4.56(2 \mathrm{H}, \mathrm{s}), 7.14(1 \mathrm{H}, \mathrm{m}), 7.35(3 \mathrm{H}, \mathrm{m}), 7.51(1 \mathrm{H}, \mathrm{m}), 7.76(2 \mathrm{H}, \mathrm{m})$, $8.65(1 \mathrm{H}, \mathrm{d}, J=3.4 \mathrm{~Hz}) ;{ }^{13} \mathrm{C}-\mathrm{NMR}\left(300 \mathrm{MHz}, \mathrm{CDCl}_{3}\right): \delta 21.4,50.4,52.9(2 \mathrm{C}), 54.3,103.4,122.7$, 127.1 (2C), 127.3, 128.7, 129.5, 129.6 (2C), 132.4, 135.8, 143.4, 149.6; MS m/z (CI): $430\left(\mathrm{MH}^{+}\right.$, $\left.{ }^{81} \mathrm{Br}, 10 \%\right), 428\left(\mathrm{MH}^{+},{ }^{79} \mathrm{Br}, 11 \%\right), 398$ (70), 396 (71) (Calcd. for: $\mathrm{C}_{18} \mathrm{H}_{22}{ }^{79} \mathrm{BrNO}_{4} \mathrm{~S}: \mathrm{M}, 427.0453$. HRMS found: $\left.\mathrm{M}^{+}, 427.0444\right)$.

8-Bromoisoquinoline (5). The benzylamino acetal $2(5.8 \mathrm{~g}, 21 \mathrm{mmol})$ was added to a solution of phosphorus pentoxide in sulphuric acid, at $0 \mathrm{C}$. The mixture was stirred for $30 \mathrm{~min}$ at this temperature then poured onto ice and the resulting solution made alkaline with sodium carbonate, and extracted with $\mathrm{CH}_{2} \mathrm{Cl}_{2}$, dried, and evaporated under reduced pressure to give a brown oil. Purification by column chromatography over silica gel eluting with $\mathrm{CH}_{2} \mathrm{Cl}_{2}$ gave pure 8bromoisoquinoline $5(146 \mathrm{mg}, 4 \%)$ as a brown solid, mp 77-79 C (lit $\left.{ }^{3}: 79-81 \mathrm{C}\right)$; ${ }^{1} \mathrm{H}-\mathrm{NMR}(300$ $\left.\mathrm{MHz}_{\mathrm{CDCl}}\right): \delta 7.56(1 \mathrm{H}, \mathrm{t}, J=7.5 \mathrm{~Hz}), 7.66(1 \mathrm{H}, \mathrm{d}, J=5.6 \mathrm{~Hz}), 7.85(2 \mathrm{H}, \mathrm{m}), 8.64(1 \mathrm{H}, \mathrm{d}$, $J=5.6 \mathrm{~Hz}), 9.63(1 \mathrm{H}, \mathrm{s}) ;{ }^{13} \mathrm{C}-\mathrm{NMR}\left(300 \mathrm{MHz}, \mathrm{CDCl}_{3}\right): \delta 120.0,122.2,126.4,130.6,131.2,136.9$, 137.1, 143.8, 161.9; MS m/z (CI): $210\left(\mathrm{MH}^{+},{ }^{81} \mathrm{Br}, 98 \%\right), 208\left(\mathrm{MH}^{+},{ }^{79} \mathrm{Br}, 100 \%\right), 130$ (75) (Calcd. for: $\mathrm{C}_{9} \mathrm{H}_{6}{ }^{79} \mathrm{BrN}$ : M, 206.9684; HRMS found: $\left.\mathrm{M}^{+}, 206.9680\right)$.

2-Bromo-3-methoxybenzaldehyde. To a solution of $N, N, N$ '-trimethylethylenediamine (4.1 ml, 32 $\mathrm{mmol})$ in benzene $(80 \mathrm{ml})$ was added $n-\mathrm{BuLi}(19 \mathrm{ml}, 1.6 \mathrm{M}, 30 \mathrm{mmol})$ dropwise with cooling. After $15 \mathrm{~min}$ at room temperature, $m$-anisaldehyde $(3.7 \mathrm{ml}, 30 \mathrm{mmol})$ was added at $0 \mathrm{C}$, and the mixture was stirred at room temperature for $15 \mathrm{~min}$. A solution of phenyllithium $(50 \mathrm{ml}, 1.8 \mathrm{M}, 90 \mathrm{mmol})$ in cyclohexane/ether was added with cooling. After the mixture was stirred at room temperature for 6 hours, THF $(65 \mathrm{ml})$ was added and the mixture cooled to $-78 \mathrm{C}$. 1,2-Dibromotetrafluoroethane $(8$ $\mathrm{ml}, 67 \mathrm{mmol}$ ) was added slowly at $-78 \mathrm{C}$, the cooling bath was removed, and the mixture was allowed to come to $\mathrm{rt}$ and stirred for $30 \mathrm{~min}$. The mixture was poured into cold water, vigorously stirred while $10 \%$ aq. $\mathrm{HCl}$ was added, and product extracted into ether. The combined organic layers were washed with brine, dried, and evaporated under reduced pressure to give an orange oil. Purification by column chromatography over silica eluting with hexane-EtOAc (92:8). The resulting material was recrystallised from hexane, giving 2-bromo-3-methoxybenzaldehyde $(5.1 \mathrm{~g}, 79 \%)$ as a white crystalline solid, mp 66-68 ${ }^{\circ} \mathrm{C}$ (lit $\left.{ }^{9}: 69-70 \mathrm{C}\right)$; IR ( NaCl): $\operatorname{vmax} 1692 \mathrm{~cm}^{-1} ;{ }^{1} \mathrm{H}-\mathrm{NMR}(300 \mathrm{MHz}$, $\left.\mathrm{CDCl}_{3}\right): \delta 3.97(3 \mathrm{H}, \mathrm{s}), 7.15(1 \mathrm{H}, \mathrm{d}, J=7.8 \mathrm{~Hz}), 7.40(1 \mathrm{H}, \mathrm{t}, J=7.8 \mathrm{~Hz}), 7.53(1 \mathrm{H}, \mathrm{d}, J=7.8 \mathrm{~Hz})$, $10.45(1 \mathrm{H}, \mathrm{s}) ;{ }^{13} \mathrm{C}-\mathrm{NMR}\left(300 \mathrm{MHz}, \mathrm{CDCl}_{3}\right): \delta 56.5,116.8,117.0,121.3,128.8,134.7,156.2,192.1$; MS m/z (EI): $216\left(\mathrm{M}^{+},{ }^{81} \mathrm{Br}, 85 \%\right), 214\left(\mathrm{M}^{+},{ }^{79} \mathrm{Br}, 100\right)$ (Calcd. for: $\mathrm{C}_{8} \mathrm{H}_{7}{ }^{79} \mathrm{BrO}_{2}$ : M, 213.9629; HRMS found: $\mathrm{M}^{+}, 213.9626$. Anal. Calcd. for: $\mathrm{C}, 44.68$; H, 3.28; Br, 37.16\%; found: C, 45.08; $\mathrm{H}$, $3.00 ; \mathrm{Br}, 36.62 \%)$.

N-(2-Bromo-3-methoxybenzylidene)aminoacetaldehyde dimethyl acetal (6). 2-Bromo-3methoxybenzaldehyde (3 g, $13.9 \mathrm{mmol})$ was dissolved in dry toluene $(45 \mathrm{ml})$ and aminoacetaldehyde dimethyl acetal $(1.6 \mathrm{ml}, 14.6 \mathrm{mmol})$ was added. The solution was refluxed 
under a Dean-Stark trap for $4 \mathrm{~h}$. Removal of the solvent in vacuum gave the benzylidene derivative $6(4.14 \mathrm{~g}, 98 \%)$ as a yellow oil; IR $(\mathrm{NaCl}):{ }_{\text {vmax }} 1639 \mathrm{~cm}^{-1} ;{ }^{1} \mathrm{H}-\mathrm{NMR}\left(300 \mathrm{MHz}, \mathrm{CDCl}_{3}\right): \delta 3.46$ $(6 \mathrm{H}, \mathrm{s}), 3.86(2 \mathrm{H}, \mathrm{dd}, J=1.4$ and $5.3 \mathrm{~Hz}), 3.95(3 \mathrm{H}, \mathrm{s}), 4.73(1 \mathrm{H}, \mathrm{t}, J=5.3 \mathrm{~Hz}), 6.98(1 \mathrm{H}, \mathrm{dd}, J=1.5$ and $8.1 \mathrm{~Hz}), 7.33(1 \mathrm{H}, \mathrm{d}, J=7.7 \mathrm{~Hz}), 7.65(1 \mathrm{H}, \mathrm{dd}, \mathrm{J}=1.5$ and $7.7 \mathrm{~Hz}), 8.78(1 \mathrm{H}, \mathrm{bs}) ;{ }^{13} \mathrm{C}-\mathrm{NMR}(300$ $\left.\mathrm{MHz} \mathrm{CDCl}_{3}\right): \delta 53.9,56.4,63.3,103.6,113.4,114.9,120.7,127.8,135.9,155.8,162.8 ; \mathrm{MS} \mathrm{m} / \mathrm{z}$ (CI): $304\left(\mathrm{MH}^{+},{ }^{81} \mathrm{Br}, 100 \%\right), 302\left(\mathrm{MH}^{+},{ }^{79} \mathrm{Br}, 95 \%\right)$ (Calcd. for: $\mathrm{C}_{12} \mathrm{H}_{16}{ }^{79} \mathrm{BrNO}_{3}+\mathrm{H}: 302.0392$; HRMS found: $\left.(\mathrm{M}+\mathrm{H})^{+}, 302.0386\right)$.

$N$-(2-Bromo-3-methoxybenzyl)aminoacetaldehyde dimethyl acetal (7) and $N$-(2-bromo-3methoxybenzyl)aminoacetaldehyde dimethyl acetal borane (17). To a solution of $\mathrm{N}$-(2-bromo-3methoxybenzylidene)aminoacetaldehyde dimethyl acetal $6(4.14 \mathrm{~g}, 13.7 \mathrm{mmol})$ in a mixture of THF $(26 \mathrm{ml})$, isopropanol $(26 \mathrm{ml})$, and water $(13 \mathrm{ml})$ was added $\mathrm{NaBH}_{4}(3.1 \mathrm{~g}, 82.2 \mathrm{mmol})$ and the mixture stirred at $\mathrm{rt}$ for $2 \mathrm{~h}$. The organic solvents were evaporated in vacuo. Addition of water and $\mathrm{CH}_{2} \mathrm{Cl}_{2}$ followed by separation, drying, and evaporation of the organic phase under pressure gave a yellow oil. Purification by column chromatography over silica gel eluting with $\mathrm{CH}_{2} \mathrm{Cl}_{2}$ gave the pure amine-borane $17(740 \mathrm{~g}, 17 \%)$ as a white crystalline solid, mp 102-105 ${ }^{\circ} \mathrm{C}$; IR ( $\left.\mathrm{NaCl}\right):{ }^{\mathrm{max}}$ $2371 \mathrm{~cm}^{-1} ;{ }^{1} \mathrm{H}-\mathrm{NMR}\left(300 \mathrm{MHz}, \mathrm{CDCl}_{3}\right): \delta 2.65(1 \mathrm{H}, \mathrm{m}), 2.71(1 \mathrm{H}, \mathrm{m}), 3.33(3 \mathrm{H}, \mathrm{s}), 3.35(3 \mathrm{H}, \mathrm{s})$, $3.72(1 \mathrm{H}, \mathrm{dd}, \mathrm{J}=9.8$ and $13.8 \mathrm{~Hz}), 3.89(3 \mathrm{H}, \mathrm{s}), 4.29(1 \mathrm{H}, \mathrm{dd}, \mathrm{J}=3.8$ and $13.8 \mathrm{~Hz}), 4.38(1 \mathrm{H}, \mathrm{bs})$, $4.79(1 \mathrm{H}, \mathrm{dd}, \mathrm{J}=3.8$ and $8.1 \mathrm{~Hz}), 6.91(2 \mathrm{H}, \mathrm{m}), 7.29(1 \mathrm{H}, \mathrm{m}) ;{ }^{13} \mathrm{C}-\mathrm{NMR}\left(300 \mathrm{MHz}, \mathrm{CDCl}_{3}\right): \delta 54.0$, 55.3, 55.6, 56.3, 60.7, 110.1, 112.4, 113.8, 124.7, 128.4, 133.3, 156.3; MS m/z (CI): $318\left(\mathrm{MH}^{+}\right.$, $\left.{ }^{81} \mathrm{Br}, 87 \%\right), 316\left(\mathrm{MH}^{+},{ }^{79} \mathrm{Br}, 100 \%\right.$ ) (Calcd. for: $\mathrm{C}_{12} \mathrm{H}_{21} \mathrm{~B}^{79} \mathrm{BrNO}_{3}$ : M, 317.0798; HRMS found: $\mathrm{M}^{+}$, 317.0795. Anal. calcd. for $\mathrm{C}_{12} \mathrm{H}_{21} \mathrm{~B}^{79} \mathrm{BrNO}_{3}$ : C, 45.32; $\mathrm{H}, 6.66 ; \mathrm{N}, 4.40 \%$; found: $\mathrm{C}, 45.01 ; \mathrm{H}, 6.54$; $\mathrm{N}, 4.18 \%)$. Elution with $\mathrm{CH}_{2} \mathrm{Cl}_{2} / \mathrm{MeOH}(9: 1)$ gave the amine $7(2.8 \mathrm{~g}, 69 \%)$ as a colorless oil; ${ }^{1} \mathrm{H}-$ NMR (300 MHz, CDCl $): \delta 2.77(2 \mathrm{H}, \mathrm{d}, J=5.6 \mathrm{~Hz}), 3.39(6 \mathrm{H}, \mathrm{s}), 3.92(3 \mathrm{H}, \mathrm{s}), 3.94(2 \mathrm{H}, \mathrm{s}), 4.50$ $(1 \mathrm{H}, \mathrm{t}, J=5.6 \mathrm{~Hz}), 6.85(1 \mathrm{H}, \mathrm{dd}, J=1.2$ and $8.1 \mathrm{~Hz}), 7.02(1 \mathrm{H}, \mathrm{dd}, J=1.2$ and $7.0 \mathrm{~Hz}), 7.28(1 \mathrm{H}, \mathrm{d}$, $J=7.0 \mathrm{~Hz}) ;{ }^{13} \mathrm{C}-\mathrm{NMR}\left(300 \mathrm{MHz}, \mathrm{CDCl}_{3}\right): \delta 50.1,53.7,53.8,56.2,103.7,110.5,113.2,122.1,127.7$, 140.7, 155.9; MS m/z (CI): $306\left(\mathrm{MH}^{+},{ }^{81} \mathrm{Br}, 90 \%\right), 304\left(\mathrm{MH}^{+},{ }^{79} \mathrm{Br}, 100 \%\right)$ (Calcd. for: $\mathrm{C}_{12} \mathrm{H}_{18}{ }^{79} \mathrm{BrNO}_{3}$ : M, 303.0470; HRMS found: $\left.\mathrm{M}^{+}, 303.0468\right)$.

A crystal of 17 of approximate dimensions $0.40 \times 0.20 \times 0.15 \mathrm{~mm}$ suitable for X-ray crystallography was prepared by recrystallisation from hexane. Data were obtained using a Rigaku AFC-5R diffractometer, graphite monochromated $\mathrm{Cu}-\mathrm{Ka}$ radiation, and a rotating anode generator. Crystal data for 17: colourless, prismatic crystals, triclinic, space group $P 1, M=318.02 ; V=$ $1562.5(4) \AA^{3} ; a=14.290(7), b=13.656(8), c=8.936(7) \AA ; Z=4 ; D_{\mathrm{c}}=1.352 \mathrm{~g} \mathrm{~cm}^{-3} ; h,-17 囚 18, k-$ $17 \AA 17, l,-11 \circledR 11 ; R=0.0442$.

$\boldsymbol{N}$-(2-Bromo-3-methoxybenzyl)- $\boldsymbol{N}$-(4-methylphenylsulfonyl)aminoacetaldehyde dimethyl acetal (8). Benzylamino-acetal $7(1.7 \mathrm{~g}, 5.6 \mathrm{mmol})$ was dissolved in dry pyridine $(7 \mathrm{ml})$ and tosyl chloride $(1.2 \mathrm{~g}, 6.2 \mathrm{mmol})$ in dry pyridine $(7 \mathrm{ml})$ added. The solution was stirred at $\mathrm{rt}$ for $24 \mathrm{~h}$. The precipitated pyridine hydrochloride was filtered off and the filtrate poured into water and product extracted into ether. The ether extract was washed with $1 \mathrm{M}$ aq. $\mathrm{HCl}$ and water. The organic layer was separated, dried, and evaporated under reduced pressure to give the pure tosylamide 8 ( $2.4 \mathrm{~g}$, 93\%) as a yellow oil; ${ }^{1} \mathrm{H}-\mathrm{NMR}\left(300 \mathrm{MHz}, \mathrm{CDCl}_{3}\right): \delta 2.42(3 \mathrm{H}, \mathrm{s}), 3.24(6 \mathrm{H}, \mathrm{s}) 3.34(2 \mathrm{H}, \mathrm{d}, J=5.3$ $\mathrm{Hz}), 3.92(3 \mathrm{H}, \mathrm{s}), 4.39(1 \mathrm{H}, \mathrm{t}, J=5.3 \mathrm{~Hz}), 4.60(2 \mathrm{H}, \mathrm{s}), 6.85(1 \mathrm{H}, \mathrm{d}, J=8.1 \mathrm{~Hz}), 7.13(1 \mathrm{H}, \mathrm{d}, J=7.7$ $\mathrm{Hz}), 7.28(1 \mathrm{H}, \mathrm{m}), 7.35(2 \mathrm{H}, \mathrm{d}, J=8.3 \mathrm{~Hz}), 7.78(2 \mathrm{H}, \mathrm{d}, J=8.3 \mathrm{~Hz}) ;{ }^{13} \mathrm{C}-\mathrm{NMR}\left(300 \mathrm{MHz}, \mathrm{CDCl}_{3}\right): \delta$ 21.4, 50.3, 53.1, 54.2, 56.3, 103.2, 110.5, 121.5, 127.1 (2C), 127.7, $129.6(2 \mathrm{C}), 133.5,137.5,143.9$, 155.7; MS m/z (CI): 396 (97\%), 394 (100\%) (Calcd. for: $\mathrm{C}_{19} \mathrm{H}_{24}{ }^{79} \mathrm{BrNO}_{5} \mathrm{~S}+\mathrm{H}: \mathrm{M}, 458.0637$; HRMS found: $\left.(\mathrm{M}+\mathrm{H})^{+}, 458.0642\right)$. 


\section{8-Bromo-3-methoxyisoquinoline 9 and 8-bromo-3-(8-bromo-7-methoxyisoquinolin-4-yl)-} 1,2,3,4-tetrahydro-7-methoxy-2-(4-methylphenylsulfonyl)isoquinoline (10). Acetal 8 (1.7g, $3.7 \mathrm{mmol}$ ) was added to a solution of $p$-toluensulfonic acid (380 $\mathrm{mg}, 2 \mathrm{mmol})$ in dry toluene (44 ml) and the mixture was stirred for $30 \mathrm{~min}$ at reflux. The mixture was made alkaline with sodium carbonate, extracted with $\mathrm{CH}_{2} \mathrm{Cl}_{2}$, dried, and evaporated under reduced pressure to give a brown oil. Purification by column hromatography over silica gel eluting with $\mathrm{CH}_{2} \mathrm{Cl}_{2}$ /EtOAc (93:4) gave the 'dimer' 10 (237 mg, 27\%) as white solid mp 253-255 C (from MeOH); ${ }^{1} \mathrm{H}-\mathrm{NMR}(300 \mathrm{MHz}$, $\left.\mathrm{CDCl}_{3}\right): \delta 2.42(3 \mathrm{H}, \mathrm{s}), 3.27(1 \mathrm{H}, \mathrm{dd}, J=7.8$ and $11.9 \mathrm{~Hz}), 3.87(1 \mathrm{H}, \mathrm{m}), 3.90(3 \mathrm{H}, \mathrm{s}), 4.10(3 \mathrm{H}, \mathrm{s})$, $4.25(1 \mathrm{H}, \mathrm{d}, J=16.1 \mathrm{~Hz}), 4.73(1 \mathrm{H}, \mathrm{d}, J=16.1 \mathrm{~Hz}), 5.00(1 \mathrm{H}, \mathrm{bs}), 6.72(1 \mathrm{H}, \mathrm{d}, J=8.5 \mathrm{~Hz}), 6.76(1 \mathrm{H}$, d, $J=8.5 \mathrm{~Hz}), 7.25(2 \mathrm{H}, \mathrm{d}, J=8.1 \mathrm{~Hz}), 7.50(1 \mathrm{H}, \mathrm{d}, J=9.2 \mathrm{~Hz}), 7.62(2 \mathrm{H}, \mathrm{d}, J=8.1 \mathrm{~Hz}), 7.86(1 \mathrm{H}, \mathrm{d}$, $J=9.2 \mathrm{~Hz}), 8.10(1 \mathrm{H}, \mathrm{s}), 9.50(1 \mathrm{H}, \mathrm{s}) ;{ }^{13} \mathrm{C}-\mathrm{NMR}\left(300 \mathrm{MHz}, \mathrm{CDCl}_{3}\right): \delta 21.4,48.9,49.0,56.3,57.0$, 109. 2, 110.7, 111.4, 117.9, 123.3, 127.4 (2C), 128.8, 129.4, 129.6 (2C), 130.1, 133.1, 136.5, 138.9, 139.2, 143.8, 145.5, 154.3, 154.7; MS m/z (EI): 630, 632, $634\left(\mathrm{M}^{+}, 20,30,20 \%\right)$ (Calcd. For: $\mathrm{C}_{27} \mathrm{H}_{24}{ }^{79} \mathrm{Br}_{2} \mathrm{~N}_{2} \mathrm{O}_{4} \mathrm{~S}$ : M, 629.9824; HRMS found: $\mathrm{M}^{+}$, 629.9837. Anal. Calcd. For: $\mathrm{C}_{27} \mathrm{H}_{24} \mathrm{Br}_{2} \mathrm{~N}_{2} \mathrm{O}_{4} \mathrm{~S}$ : C, 51.28; H, 3.83; N, 4.43; Br, 25.27; S, 5.07\%; found: C, 51.17; H, 3.80; N, 4.39; Br, 25.02; S, 5.24\%). Further elution gave 8-bromo-3-methoxyisoquinoline 9 (168 $\mathrm{mg}, 19 \%)$ as a yellow solid, mp 105-107 C (lit : 108-109 $\left.{ }^{\circ} \mathrm{C}\right) ;{ }^{1} \mathrm{H}-\mathrm{NMR}\left(300 \mathrm{MHz}, \mathrm{CDCl}_{3}\right): \delta 4.08(3 \mathrm{H}, \mathrm{s}), 7.50(1 \mathrm{H}, \mathrm{d}, J=9.0$ $\mathrm{Hz}), 7.59(1 \mathrm{H}, \mathrm{d}, J=5.6 \mathrm{~Hz}), 7.84(1 \mathrm{H}, \mathrm{d}, J=9.0 \mathrm{~Hz}), 8.52(1 \mathrm{H}, \mathrm{d}, J=5.6 \mathrm{~Hz}), 9.62(1 \mathrm{H}, \mathrm{s}) ;{ }^{13} \mathrm{C}-\mathrm{NMR}$ $\left(300 \mathrm{MHz}, \mathrm{CDCl}_{3}\right): \delta 57.0,108.1,117.8,119.6,127.6,127.9,132.1,141.5,151.0,154.1 ; \mathrm{MS} \mathrm{m} / \mathrm{z}$ (EI): $239\left(\mathrm{M}^{+},{ }^{81} \mathrm{Br}, 98 \%\right.$ ), $237\left(\mathrm{M}^{+},{ }^{79} \mathrm{Br}, 100 \%\right.$ (Calcd. For: $\mathrm{C}_{10} \mathrm{H}_{8}{ }^{79} \mathrm{BrNO}$ : M, 236.9789; HRMS found: $\left.\mathrm{M}^{+}, 236.9793\right)$.

8-(Hex-1-yn-1-yl)-7-methoxyisoquinoline (15). A mixture of 8-bromo-7-methoxyisoquinoline 9 (375 mg, $1.5 \mathrm{mmol})$, 1-hexyne $(0.17 \mathrm{ml}, 3 \mathrm{mmol})$, bis(triphenyl phosphine)palladium(II) chloride(32 mg, $0.045 \mathrm{mmol})$, copper(I) iodide (17 mg, $0.09 \mathrm{mmol}$ ) and triethylamine (4.5 ml) was heated at $80{ }^{\circ} \mathrm{C}$ overnight under nitrogen. The residue was diluted with water and extracted with dichloromethane and the organic extract was washed with $1 \mathrm{~N}$-hydrochloric acid. Separation, drying and evaporation of the organic phase under reduced pressure gave a brown oil. Purification by column chromatography over silica gel eluting with petroleum ether/EtOAc gave 8-(hex-1-yn-1-yl)7-methoxyisoquinoline $15(135 \mathrm{mg}, 37 \%)$ as a yellow oil; ${ }^{1} \mathrm{H}-\mathrm{NMR}\left(300 \mathrm{MHz}, \mathrm{CDCl}_{3}\right): \delta 1.02(3 \mathrm{H}$, t, $J=7.2 \mathrm{~Hz}), 1.62(2 \mathrm{H}, \mathrm{m}), 1.75(2 \mathrm{H}, \mathrm{m}), 2.68(2 \mathrm{H}, \mathrm{t}, J=7.1 \mathrm{~Hz}), 4.06(3 \mathrm{H}, \mathrm{s}), 7.47(1 \mathrm{H}, \mathrm{d}, J=9.1$ $\mathrm{Hz}), 7.60(1 \mathrm{H}, \mathrm{bs}), 7.76(1 \mathrm{H}, \mathrm{d}, J=9.1 \mathrm{~Hz}), 8.50(1 \mathrm{H}, \mathrm{bs}), 9.67(1 \mathrm{H}, \mathrm{bs}) ;{ }^{13} \mathrm{C}-\mathrm{NMR}(300 \mathrm{MHz}$, $\left.\mathrm{CDCl}_{3}\right): \delta 12.9,19.7,22.0,30.8,56.7,117.7,127.4,129.7,141.3,150.5 ; \mathrm{MS} \mathrm{m} / \mathrm{z}(\mathrm{CI}): 240(\mathrm{M}+\mathrm{H}$, $100 \%$ ). Calcd. for: $\mathrm{C}_{16} \mathrm{H}_{17} \mathrm{NO}: \mathrm{M}, 239.1310$; HRMS found: $\mathrm{M}^{+}, 239.1315$.

$\boldsymbol{N}$-(2-Bromobenzylidene)ethanamine (18). 2-Bromobenzaldehyde (2.5 ml, $13.15 \mathrm{mmol})$ was dissolved in toluene $(45 \mathrm{ml})$ and ethanamine solution $(70 \%$ aq., $0.81 \mathrm{ml}, 14.4 \mathrm{mmol})$ was added. The mixture was refluxed under a Dean-Stark trap for 4 hours. The solution was evaporated in vacuum to give the $N$-(2-bromobenzylidene)ethyl amine $18(2.2 \mathrm{~g}, 79 \%)$ as an orange oil; ${ }^{1} \mathrm{H}-\mathrm{NMR}$ $\left(300 \mathrm{MHz}, \mathrm{CDCl}_{3}\right): \delta 1.36(3 \mathrm{H}, \mathrm{t}, J=7.3 \mathrm{~Hz}), 3.74(2 \mathrm{H}, \mathrm{q}, J=7.3 \mathrm{~Hz}), 7.34(2 \mathrm{H}, \mathrm{m}), 7.60(1 \mathrm{H}, \mathrm{d}, J=$ $7.8 \mathrm{~Hz}), 8.05(1 \mathrm{H}, \mathrm{dd}, \mathrm{J}=7.7 \mathrm{~Hz}), 8.70(1 \mathrm{H}, \mathrm{s}, 1 \mathrm{H}) ;{ }^{13} \mathrm{C}-\mathrm{NMR}\left(300 \mathrm{MHz}, \mathrm{CDCl}_{3}\right): \delta 16.1,55.8$, 124.7, 127.5, 128.6, 131.5, 132.8, 134. 2 159.4; MS m/z (CI): $214\left(\mathrm{MH}^{+},{ }^{81} \mathrm{Br}, 90 \%\right), 212\left(\mathrm{MH}^{+}\right.$, ${ }^{79} \mathrm{Br}, 100 \%$ ) (Calcd. for: $\mathrm{C}_{9} \mathrm{H}_{10}{ }^{79} \mathrm{BrN}, \mathrm{M}: 210.9997$; HRMS found: $\mathrm{M}^{+}, 210.9995$ ).

$\boldsymbol{N}$-(2-Bromobenzyl)ethanamine (19) and $\boldsymbol{N}$-(2-bromobenzyl)ethanamine borane (20). To a solution of $N$-(2-bromobenzylidene)ethanamine $(1 \mathrm{~g}, 4.71 \mathrm{mmol})$ in a mixture of THF $(10 \mathrm{ml})$, isopropanol $(10 \mathrm{ml})$, and water $(5 \mathrm{ml})$ was added $\mathrm{NaBH}_{4}(1.07 \mathrm{~g}, 28.2 \mathrm{mmol})$ and the reaction mixture stirred at $\mathrm{rt}$ for $2 \mathrm{~h}$. The organic solvents were evaporated in vacuum. Addition of water and 
$\mathrm{CH}_{2} \mathrm{Cl}_{2}$ followed by separation, drying, and evaporation of the organic phase under reduced pressure gave a yellow oil. Purification by column chromatography over silica gel eluting with $\mathrm{CH}_{2} \mathrm{Cl}_{2}$ gave the pure amine-borane $20(220 \mathrm{mg}, 20 \%)$ as a colourless oil; IR $(\mathrm{NaCl}): v \operatorname{vmax} 2372 \mathrm{~cm}^{-}$ ${ }^{1} ;{ }^{1} \mathrm{H}-\mathrm{NMR}\left(300 \mathrm{MHz}, \mathrm{CDCl}_{3}\right): \delta 1.18(3 \mathrm{H}, \mathrm{t}, J=7.2), 2.70(2 \mathrm{H}, \mathrm{m}), 3.80(2 \mathrm{H}, \mathrm{m}), 4.12(1 \mathrm{H}, \mathrm{dd}$, $J=4.6$ and $12.6 \mathrm{~Hz}), 7.24(3 \mathrm{H}, \mathrm{m}), 7.58(1 \mathrm{H}, \mathrm{d}, \mathrm{J}=7.7 \mathrm{~Hz}) ;{ }^{13} \mathrm{C}-\mathrm{NMR}\left(300 \mathrm{MHz}, \mathrm{CDCl}_{3}\right): \delta 11.7$, 48.4, 59.6, 124.2, 127.8, 130.6, 133.1, 133.2, 134.3; MS m/z (EI): $228\left(\mathrm{M}^{+},{ }^{81} \mathrm{Br}, 50 \%\right), 226\left(\mathrm{M}^{+}\right.$, ${ }^{79} \mathrm{Br}$, 40\%) (Calcd. for: $\mathrm{C}_{9} \mathrm{H}_{15} \mathrm{~B}^{79} \mathrm{BrN}$ : M, 245.0825; HRMS found: 245.0824). Elution with $\mathrm{CH}_{2} \mathrm{Cl}_{2} / \mathrm{MeOH}(9: 1)$ gave the amine $19(683 \mathrm{mg}, 68 \%)$ as a colorless oil; ${ }^{1} \mathrm{H}-\mathrm{NMR}$ (300 $\mathrm{MHz}$, $\left.\mathrm{CDCl}_{3}\right): \delta 1.18(3 \mathrm{H}, \mathrm{t}, J=7.1), 2.73(2 \mathrm{H}, \mathrm{q}, J=7.1 \mathrm{~Hz}), 3.90(2 \mathrm{H}, \mathrm{s}), 7.15(1 \mathrm{H}, \mathrm{t}, J=7.7 \mathrm{~Hz}), 7.32(1 \mathrm{H}$, m), $7.43(1 \mathrm{H}, \mathrm{d}, J=7.7 \mathrm{~Hz}), 7.58(1 \mathrm{H}, \mathrm{d}, J=7.9 \mathrm{~Hz}) ;{ }^{13} \mathrm{C}-\mathrm{NMR}\left(300 \mathrm{MHz}, \mathrm{CDCl}_{3}\right): \delta 15.2,43.3$, 53.5, 123.8, 127.3, 128.4, 130.1, 132.6, 139.2; $\mathrm{MS} \mathrm{m} / \mathrm{z}(\mathrm{CI}): 216\left(\mathrm{MH}^{+},{ }^{81} \mathrm{Br}, 100\right), 214\left(\mathrm{MH}^{+},{ }^{79} \mathrm{Br}\right.$, 98\%) (Calcd. for: $\mathrm{C}_{9} \mathrm{H}_{12}{ }^{79} \mathrm{BrN}$ : M, 213.0153; HRMS found: $\mathrm{M}^{+}$, 213.0157).

\section{General procedure for reaction of secondary amines with $\mathrm{BH}_{3}$ - THF}

To a solution of the amine $(1 \mathrm{mmol})$ in THF $(5 \mathrm{ml}), \mathrm{BH}_{3}$-THF $(1 \mathrm{M}, 2.6 \mathrm{mmol})$ was added. The mixture was stirred at $\mathrm{rt}$ for $15 \mathrm{~min}$. The solvent was evaporated to dryness to leave the amineborane in quantitative yield.

\section{Acknowledgements}

We are greatly indebted to the Nissan Chemical Company, Japan for their interest in and support for this work and for providing a studentship (MA), and to the SERC for funds for the purchase of the Rigaku AFC-5R diffractometer.

\section{References}

1. Claus, Hoffmann, K. J. prakt. Chem. 1893, 47, 252.

2. Crawford, M.; Smyth, I. F. B. J. Chem. Soc. 1954, 3464.

3. Glyde, E.; Taylor, R. J. Chem. Soc., Perkin Trans. 2, 1975, 1783.

4. Birch, J.; Jackson, A. H.; Shannon, P. V. R. J. Chem. Soc., Perkin Trans. 1, 1974, 2185.

5. Popp, F. D.; Buhts, R. E.; Chesney, D. K. J. Heterocycl. Chem. 1978, 15, 429.

6. Ronne, E.; Grivas, S. Heterocycles 1993, 36, 101.

7. Ahmad, Y.; Hey, D. H. J. Chem. Soc. 1961, 3882.

8. Bohlmann, F.; Albrecht, K.-D.; Schmidt, G. Chem. Ber. 1966, 99, 2822.

9. Moorlag, H.; Meyers, A. I. Tetrahedron Lett. 1993, 34, 6993.

10. Carboni, B.; Monnier, L. 'Recent developments in the chemistry of amine- and phosphineboranes', Tetrahedron 1999, 55, 1197.

11. For crystal structures of cyclic secondary amine-boranes see: (a) Ringertz, H. Acta Chem. Scand. 1969, 23, 137. (b) Dupart, J.-M.; Grand, A.; Pace, S.; Riess, J. G. Inorg. Chem. 1984, 23, 3776. (c) Khasnis, D. V.; Lattman, M.; Siriwardane, U. J. Chem. Soc., Chem. Commun. 1989, 1538. (d) Jackson, T. W.; Kojima, M.; Lambrecht, R. M.; Marubayashi, N.; Hiratake, M. Aust. J. Chem. 1994, 47, 2271. 